Noncentrosymmetric Fluorooxoborates $A_{10} B_{13} O_{15} F_{19}(A=\quad K$ and $R b)$ with Unexpected $\left[\mathrm{B}_{10} \mathrm{O}_{12} \mathrm{~F}_{13}\right]^{7-}$ Units and Deep-Ultraviolet Cut-off Edges $\dagger$

Wenyao Zhang, ${ }^{\text {a, b }}$ Zhonglei Wei, ${ }^{\text {a, b }}$ Zhihua Yang, ${ }^{\text {a Shilie Pan*a }}$

${ }^{a}$ CAS Key Laboratory of Functional Materials and Devices for Special Environments, Xinjiang Technical Institute of Physics \& Chemistry, CAS, and Xinjiang Key Laboratory of Electronic Information Materials and Devices, 40-1 South Beijing Road, Urumqi 830011, China

${ }^{\mathrm{b}}$ Center of Materials Science and Optoelectronics Engineering, University of Chinese Academy of Sciences, Beijing 100049, China

*To whom correspondence should be addressed. Phone: (+86)991-3674558.

Fax: (+86)991-3838957. E-mail: slpan@ms.xjb.ac.cn (S. Pan). 


\section{Stucture refinment}

The structure determination and refinement of $\mathrm{K}_{10} \mathrm{~B}_{13} \mathrm{O}_{15} \mathrm{~F}_{19}$ and $\mathrm{Rb}_{10} \mathrm{~B}_{13} \mathrm{O}_{15} \mathrm{~F}_{19}$ were straightforward, while disorders were found in both of them. First, the $\mathrm{F}(5)$ atom was split into two atoms, $\mathrm{F}(5 \mathrm{~A})$ and $\mathrm{F}(5 \mathrm{~B})$, with the site occupation factor 0.5 in the final refinement. Second, it is also noticed that the $\mathrm{O}(1)$ atom is located at the special positions (mirror plane) without fullfilling the geometry symmetry element. In general, according to the previous study, ${ }^{1}$ there are two possible ways to handle such case: (1) use different/lower symmetry. (2) refine the disorder about the special position. We tried to solve the two crystal structures with another space group (lower symmetry), e.g., $R 3$ and $R 32$; however, no satisfied results were obtained $\left(R_{1}>0.09, w R_{2}>0.2\right)$. Therefore, the structures were solved in the space group $R 3 m$ with disorder, and a satisfying structure model with low convergence factor $(\mathrm{R} 1=0.0492)$ can be obtained. In addition, the ratio of Data / Parameter cannot be improved after several crystal data collections, which is due to the weak collected diffraction points and many restraints (e.g. ISOR command) are adopted for light atoms owing to poor absorption corrections. 
Table S1. Atomic coordinates $\left(\times 10^{4}\right)$ and equivalent isotropic displacement parameters $\left(\AA^{2} \times 10^{3}\right)$ for $\mathrm{K}_{10} \mathrm{~B}_{13} \mathrm{O}_{15} \mathrm{~F}_{19}$. $\mathrm{U}_{\text {eq }}$ is defined as one-third of the trace of the orthogonalized $\mathrm{U}_{\mathrm{ij}}$ tensor.

\begin{tabular}{llllll}
\hline Atom & $\mathrm{x}$ & $\mathrm{y}$ & $\mathrm{z}$ & $\mathrm{U}(\mathrm{eq})$ & $\mathrm{BVS}$ [a] \\
\hline $\mathrm{K}(1)$ & $1292(1)$ & $8708(1)$ & $7252(2)$ & $36(1)$ & 1.12 \\
$\mathrm{~K}(2)$ & $1349(1)$ & $12699(2)$ & $1699(2)$ & $34(1)$ & 1.10 \\
$\mathrm{~K}(3)$ & $1291(1)$ & $8709(1)$ & $4304(2)$ & $29(1)$ & 1.15 \\
$\mathrm{~K}(4)$ & 3333 & 6667 & $6434(3)$ & $32(1)$ & 1.04 \\
$\mathrm{~B}(1)$ & $-605(5)$ & $8790(10)$ & $5813(9)$ & $19(2)$ & 3.17 \\
$\mathrm{~B}(2)$ & $3870(8)$ & $9495(12)$ & $6109(7)$ & $50(3)$ & 3.09 \\
$\mathrm{~B}(3)$ & $5620(8)$ & $11240(15)$ & $6258(13)$ & $56(4)$ & 3.09 \\
$\mathrm{~B}(4)$ & 0 & 10000 & $2640(14)$ & $24(5)$ & 3.11 \\
$\mathrm{O}(1)$ & $0(7)$ & $8989(7)$ & $2914(7)$ & $24(2)$ & 1.95 \\
$\mathrm{O}(2)$ & $-1162(6)$ & $9419(3)$ & $5873(5)$ & $23(2)$ & 2.22 \\
$\mathrm{O}(3)$ & $4506(3)$ & $9013(6)$ & $5863(6)$ & $45(2)$ & 2.23 \\
$\mathrm{O}(4)$ & $4513(7)$ & $10684(6)$ & $6251(5)$ & $60(2)$ & 2.25 \\
$\mathrm{~F}(1)$ & 0 & 10000 & $1611(7)$ & $46(3)$ & 1.06 \\
$\mathrm{~F}(2)$ & $-910(3)$ & $8179(5)$ & $4940(4)$ & $34(2)$ & 1.08 \\
$\mathrm{~F}(3)$ & $-1040(3)$ & $7920(6)$ & $6513(5)$ & $39(2)$ & 1.09 \\
$\mathrm{~F}(4)$ & $3071(5)$ & $9302(7)$ & $5408(4)$ & $85(2)$ & 1.03 \\
$\mathrm{~F}(5 \mathrm{~A})$ & $3376(11)$ & $9400(30)$ & $6969(7)$ & $51(7)$ & 1.21 \\
$\mathrm{~F}(5 \mathrm{~B})$ & $3191(10)$ & $8650(20)$ & $6908(7)$ & $39(5)$ & 0.87 \\
\hline
\end{tabular}

[a] Bond valence sums are calculated by using bond-valence theory $\left(S_{i}=\right.$ $\exp \left[\left(\mathrm{R}_{\mathrm{o}}-\mathrm{R}_{\mathrm{i}} / \mathrm{B}\right]\right.$, where $\mathrm{R}_{\mathrm{o}}$ is an empirical constant, $\mathrm{R}_{\mathrm{i}}$ is the length of bond/(in angstroms), and $\mathrm{B}=0.37$ ). 
Table S2. Atomic coordinates $\left(\times 10^{4}\right)$ and equivalent isotropic displacement parameters $\left(\AA^{2} \times 10^{3}\right)$ for $\mathrm{Rb}_{10} \mathrm{~B}_{13} \mathrm{O}_{15} \mathrm{~F}_{19}$. $\mathrm{U}_{\text {eq }}$ is defined as one-third of the trace of the orthogonalized $\mathrm{U}_{\mathrm{ij}}$ tensor.

\begin{tabular}{llllll}
\hline Atom & $\mathrm{x}$ & $\mathrm{y}$ & $\mathrm{z}$ & $\mathrm{U}(\mathrm{eq})$ & $\mathrm{BVS}^{[\mathrm{a}]}$ \\
\hline $\mathrm{Rb}(1)$ & $1286(1)$ & $8714(1)$ & $7266(1)$ & $38(1)$ & 1.14 \\
$\mathrm{Rb}(2)$ & $1352(1)$ & $12704(2)$ & $1689(1)$ & $35(1)$ & 0.75 \\
$\mathrm{Rb}(3)$ & $1294(1)$ & $8706(1)$ & $4306(1)$ & $29(1)$ & 1.16 \\
$\mathrm{Rb}(4)$ & 3333 & 6667 & $6451(3)$ & $38(1)$ & 1.02 \\
$\mathrm{~B}(1)$ & $-586(8)$ & $8827(17)$ & $5805(15)$ & $21(4)$ & 3.14 \\
$\mathrm{~B}(2)$ & $3903(14)$ & $9530(20)$ & $6125(13)$ & $54(6)$ & 3.02 \\
$\mathrm{~B}(3)$ & $5638(12)$ & $11280(20)$ & $6248(19)$ & $52(5)$ & 2.75 \\
$\mathrm{~B}(4)$ & 0 & 10000 & $2670(20)$ & $22(9)$ & 3.24 \\
$\mathrm{O}(1)$ & $-34(11)$ & $9016(12)$ & $2930(12)$ & $27(4)$ & 1.95 \\
$\mathrm{O}(2)$ & $-1130(9)$ & $9435(5)$ & $5873(8)$ & $23(3)$ & 2.20 \\
$\mathrm{O}(3)$ & $4544(5)$ & $9087(10)$ & $5896(10)$ & $43(4)$ & 2.23 \\
$\mathrm{O}(4)$ & $4517(10)$ & $10693(9)$ & $6259(8)$ & $58(2)$ & 2.07 \\
$\mathrm{~F}(1)$ & 0 & 10000 & $1684(11)$ & $35(5)$ & 1.04 \\
$\mathrm{~F}(2)$ & $-881(4)$ & $8238(9)$ & $4962(7)$ & $31(3)$ & 1.25 \\
$\mathrm{~F}(3)$ & $-1020(4)$ & $7961(8)$ & $6458(7)$ & $35(3)$ & 1.11 \\
$\mathrm{~F}(4)$ & $3166(8)$ & $9382(10)$ & $5428(6)$ & $76(3)$ & 1.03 \\
$\mathrm{~F}(5 \mathrm{~A})$ & $3408(16)$ & $9520(30)$ & $6914(13)$ & $44(10)$ & 1.27 \\
$\mathrm{~F}(5 \mathrm{~B})$ & $3252(13)$ & $8740(30)$ & $6854(11)$ & $45(8)$ & 0.99 \\
\hline & & & 69 & \\
\hline
\end{tabular}

[a] Bond valence sums are calculated by using bond-valence theory $\left(\mathrm{S}_{\mathrm{i}}=\right.$ $\exp \left[\left(\mathrm{R}_{\mathrm{o}}-\mathrm{R}_{\mathrm{i}} / \mathrm{B}\right]\right.$, where $\mathrm{R}_{\mathrm{o}}$ is an empirical constant, $\mathrm{R}_{\mathrm{i}}$ is the length of bond / (in angstroms), and $\mathrm{B}=$ $0.37)$. 
Table S3. Selected bond distances $(\AA)$ and angles (deg) for $\mathrm{K}_{10} \mathrm{~B}_{13} \mathrm{O}_{15} \mathrm{~F}_{19}$

\begin{tabular}{|c|c|c|c|}
\hline $\mathrm{K}(1)-\mathrm{F}(5 \mathrm{~A})$ & $2.559(12)$ & $\mathrm{K}(4)-\mathrm{F}(1) \# 14$ & $2.636(11)$ \\
\hline $\mathrm{K}(1)-\mathrm{F}(5 \mathrm{~A}) \# 1$ & $2.559(12)$ & $\mathrm{K}(4)-\mathrm{O}(3)$ & $2.910(8)$ \\
\hline $\mathrm{K}(1)-\mathrm{O}(2) \# 2$ & $2.600(7)$ & $\mathrm{K}(4)-\mathrm{O}(3) \# 15$ & $2.910(8)$ \\
\hline $\mathrm{K}(1)-\mathrm{F}(5 \mathrm{~B})$ & $2.695(13)$ & $\mathrm{K}(4)-\mathrm{O}(3) \# 16$ & $2.910(8)$ \\
\hline $\mathrm{K}(1)-\mathrm{F}(5 \mathrm{~B}) \# 1$ & $2.695(13)$ & $\mathrm{K}(4)-\mathrm{F}(5 \mathrm{~B})$ & 2.91(2) \\
\hline $\mathrm{K}(1)-\mathrm{O}(3) \# 3$ & $2.794(9)$ & $\mathrm{K}(4)-\mathrm{F}(5 \mathrm{~B}) \# 1$ & 2.91(3) \\
\hline $\mathrm{K}(1)-\mathrm{F}(3)$ & $3.016(4)$ & $\mathrm{K}(4)-\mathrm{F}(5 \mathrm{~B}) \# 17$ & 2.91(3) \\
\hline $\mathrm{K}(1)-\mathrm{F}(3) \# 2$ & $3.016(4)$ & $\mathrm{K}(4)-\mathrm{F}(5 \mathrm{~B}) \# 15$ & 2.91(3) \\
\hline $\mathrm{K}(1)-\mathrm{F}(4) \# 3$ & $3.043(7)$ & $\mathrm{K}(4)-\mathrm{F}(5 \mathrm{~B}) \# 16$ & 2.91(3) \\
\hline $\mathrm{K}(1)-\mathrm{F}(4) \# 4$ & $3.043(7)$ & $\mathrm{K}(4)-\mathrm{F}(5 \mathrm{~B}) \# 18$ & 2.91(3) \\
\hline $\mathrm{K}(2)-\mathrm{F}(2) \# 5$ & $2.559(7)$ & $\mathrm{B}(1)-\mathrm{O}(2)$ & $1.415(7)$ \\
\hline $\mathrm{K}(2)-\mathrm{F}(5 \mathrm{~A}) \# 6$ & $2.702(12)$ & $\mathrm{B}(1)-\mathrm{O}(2) \# 2$ & $1.415(7)$ \\
\hline $\mathrm{K}(2)-\mathrm{F}(5 \mathrm{~A}) \# 7$ & $2.702(12)$ & $\mathrm{B}(1)-\mathrm{F}(3)$ & $1.440(14)$ \\
\hline $\mathrm{K}(2)-\mathrm{F}(5 \mathrm{~B}) \# 6$ & $2.738(13)$ & $\mathrm{B}(1)-\mathrm{F}(2)$ & $1.445(13)$ \\
\hline $\mathrm{K}(2)-\mathrm{F}(5 \mathrm{~B}) \# 7$ & $2.738(13)$ & $\mathrm{B}(2)-\mathrm{F}(5 \mathrm{~A})$ & $1.379(15)$ \\
\hline $\mathrm{K}(2)-\mathrm{O}(1) \# 8$ & $2.745(9)$ & $\mathrm{B}(2)-\mathrm{O}(3)$ & $1.381(12)$ \\
\hline $\mathrm{K}(2)-\mathrm{O}(1) \# 2$ & $2.745(9)$ & $\mathrm{B}(2)-\mathrm{F}(4)$ & $1.410(11)$ \\
\hline $\mathrm{K}(2)-\mathrm{O}(4) \# 9$ & $2.851(7)$ & $\mathrm{B}(2)-\mathrm{O}(4)$ & $1.432(15)$ \\
\hline $\mathrm{K}(2)-\mathrm{O}(4) \# 10$ & $2.851(7)$ & $\mathrm{B}(2)-\mathrm{F}(5 \mathrm{~B})$ & $1.562(19)$ \\
\hline $\mathrm{K}(2)-\mathrm{F}(3) \# 5$ & $3.087(8)$ & $\mathrm{B}(3)-\mathrm{O}(4) \# 17$ & $1.317(11)$ \\
\hline $\mathrm{K}(2)-\mathrm{F}(1)$ & $3.215(3)$ & $\mathrm{B}(3)-\mathrm{O}(4)$ & $1.317(11)$ \\
\hline $\mathrm{K}(2)-\mathrm{F}(4) \# 9$ & $3.294(8)$ & $\mathrm{B}(3)-\mathrm{O}(1) \# 19$ & $1.464(17)$ \\
\hline $\mathrm{K}(3)-\mathrm{F}(4)$ & $2.674(6)$ & $\mathrm{B}(3)-\mathrm{O}(1) \# 20$ & $1.464(17)$ \\
\hline $\mathrm{K}(3)-\mathrm{F}(4) \# 1$ & $2.674(6)$ & $\mathrm{B}(4)-\mathrm{O}(1)$ & $1.443(10)^{*}$ \\
\hline $\mathrm{K}(3)-\mathrm{O}(2) \# 2$ & $2.809(7)$ & $\mathrm{B}(4)-\mathrm{O}(1) \# 2$ & $1.443(10)^{*}$ \\
\hline $\mathrm{K}(3)-\mathrm{O}(1) \# 1$ & $2.817(10)$ & $\mathrm{B}(4)-\mathrm{O}(1) \# 21$ & $1.443(10)^{*}$ \\
\hline $\mathrm{K}(3)-\mathrm{O}(1)$ & $2.817(10)$ & $\mathrm{B}(4)-\mathrm{O}(1) \# 1$ & $1.443(10)^{*}$ \\
\hline $\mathrm{K}(3)-\mathrm{F}(3) \# 11$ & $2.878(7)$ & $\mathrm{B}(4)-\mathrm{O}(1) \# 8$ & $1.443(10)^{*}$ \\
\hline $\mathrm{K}(3)-\mathrm{F}(2) \# 2$ & $2.882(3)$ & $\mathrm{B}(4)-\mathrm{O}(1) \# 22$ & $1.443(10)^{*}$ \\
\hline $\mathrm{K}(3)-\mathrm{F}(2)$ & $2.882(3)$ & $\mathrm{B}(4)-\mathrm{F}(1)$ & $1.47(2)$ \\
\hline $\mathrm{K}(3)-\mathrm{O}(4) \# 12$ & $2.977(7)$ & $\mathrm{O}(2)-\mathrm{B}(1)-\mathrm{O}(2) \# 2$ & $115.6(10)$ \\
\hline $\mathrm{K}(3)-\mathrm{O}(4) \# 13$ & $2.977(7)$ & $\mathrm{O}(2)-\mathrm{B}(1)-\mathrm{F}(3)$ & $109.8(6)$ \\
\hline $\mathrm{K}(3)-\mathrm{F}(5 \mathrm{~A}) \# 12$ & $3.40(3)$ & $\mathrm{O}(2) \# 2-\mathrm{B}(1)-\mathrm{F}(3)$ & $109.8(6)$ \\
\hline $\mathrm{K}(3)-\mathrm{F}(5 \mathrm{~A}) \# 13$ & $3.40(3)$ & $\mathrm{O}(2)-\mathrm{B}(1)-\mathrm{F}(2)$ & $108.5(7)$ \\
\hline
\end{tabular}




\begin{tabular}{llll}
\hline $\mathrm{O}(2) \# 2-\mathrm{B}(1)-\mathrm{F}(2)$ & $108.5(7)$ & $\mathrm{O}(1) \# 2-\mathrm{B}(4)-\mathrm{O}(1) \# 21$ & $148.5(18)$ \\
$\mathrm{F}(3)-\mathrm{B}(1)-\mathrm{F}(2)$ & $103.8(9)$ & $\mathrm{O}(1)-\mathrm{B}(4)-\mathrm{O}(1) \# 1$ & $57.6(7)$ \\
$\mathrm{F}(5 \mathrm{~A})-\mathrm{B}(2)-\mathrm{O}(3)$ & $125.5(15)$ & $\mathrm{O}(1) \# 2-\mathrm{B}(4)-\mathrm{O}(1) \# 1$ & $57.5(7)$ \\
$\mathrm{F}(5 \mathrm{~A})-\mathrm{B}(2)-\mathrm{F}(4)$ & $108.4(9)$ & $\mathrm{O}(1) \# 21-\mathrm{B}(4)-\mathrm{O}(1) \# 1$ & $112.9(8)$ \\
$\mathrm{O}(3)-\mathrm{B}(2)-\mathrm{F}(4)$ & $110.4(9)$ & $\mathrm{O}(1)-\mathrm{B}(4)-\mathrm{O}(1) \# 8$ & $148.5(18)$ \\
$\mathrm{F}(5 \mathrm{~A})-\mathrm{B}(2)-\mathrm{O}(4)$ & $88.7(15)$ & $\mathrm{O}(1) \# 2-\mathrm{B}(4)-\mathrm{O}(1) \# 8$ & $57.6(7)$ \\
$\mathrm{O}(3)-\mathrm{B}(2)-\mathrm{O}(4)$ & $114.0(8)$ & $\mathrm{O}(1) \# 21-\mathrm{B}(4)-\mathrm{O}(1) \# 8$ & $112.9(8)$ \\
$\mathrm{F}(4)-\mathrm{B}(2)-\mathrm{O}(4)$ & $107.1(10)$ & $\mathrm{O}(1) \# 1-\mathrm{B}(4)-\mathrm{O}(1) \# 8$ & $112.9(8)$ \\
$\mathrm{O}(3)-\mathrm{B}(2)-\mathrm{F}(5 \mathrm{~B})$ & $96.7(12)$ & $\mathrm{O}(1)-\mathrm{B}(4)-\mathrm{O}(1) \# 22$ & $112.9(8)$ \\
$\mathrm{F}(4)-\mathrm{B}(2)-\mathrm{F}(5 \mathrm{~B})$ & $105.1(8)$ & $\mathrm{O}(1) \# 2-\mathrm{B}(4)-\mathrm{O}(1) \# 22$ & $112.9(8)$ \\
$\mathrm{O}(4)-\mathrm{B}(2)-\mathrm{F}(5 \mathrm{~B})$ & $122.9(11)$ & $\mathrm{O}(1) \# 21-\mathrm{B}(4)-\mathrm{O}(1) \# 22$ & $57.6(7)$ \\
$\mathrm{O}(4) \# 17-\mathrm{B}(3)-\mathrm{O}(4)$ & $119.7(15)$ & $\mathrm{O}(1) \# 1-\mathrm{B}(4)-\mathrm{O}(1) \# 22$ & $148.5(18)$ \\
$\mathrm{O}(4) \# 17-\mathrm{B}(3)-\mathrm{O}(1) \# 19$ & $91.8(5)$ & $\mathrm{O}(1) \# 8-\mathrm{B}(4)-\mathrm{O}(1) \# 22$ & $57.5(7)$ \\
$\mathrm{O}(4)-\mathrm{B}(3)-\mathrm{O}(1) \# 19$ & $148.4(12)$ & $\mathrm{O}(1)-\mathrm{B}(4)-\mathrm{F}(1)$ & $105.7(9)$ \\
$\mathrm{O}(4) \# 17-\mathrm{B}(3)-\mathrm{O}(1) \# 20$ & $148.4(12)$ & $\mathrm{O}(1) \# 2-\mathrm{B}(4)-\mathrm{F}(1)$ & $105.7(9)$ \\
$\mathrm{O}(4)-\mathrm{B}(3)-\mathrm{O}(1) \# 20$ & $91.8(5)$ & $\mathrm{O}(1) \# 21-\mathrm{B}(4)-\mathrm{F}(1)$ & $105.7(9)$ \\
$\mathrm{O}(1) \# 19-\mathrm{B}(3)-\mathrm{O}(1) \# 20$ & $56.6(10)$ & $\mathrm{O}(1) \# 1-\mathrm{B}(4)-\mathrm{F}(1)$ & $105.7(9)$ \\
$\mathrm{O}(1)-\mathrm{B}(4)-\mathrm{O}(1) \# 2$ & $112.9(8)$ & $\mathrm{O}(1) \# 8-\mathrm{B}(4)-\mathrm{F}(1)$ & $105.7(9)$ \\
$\mathrm{O}(1)-\mathrm{B}(4)-\mathrm{O}(1) \# 21$ & $57.5(7)$ & $\mathrm{O}(1) \# 22-\mathrm{B}(4)-\mathrm{F}(1)$ & $105.7(9)$ \\
\hline
\end{tabular}

*: The $\mathrm{O}(1)$ is disordered in special site.

Symmetry transformations used to generate equivalent atoms:

\begin{tabular}{|l|l|l|}
\hline$\# 1-\mathrm{y}+1,-\mathrm{x}+1, \mathrm{z}$ & $\# 2-\mathrm{y}+1, \mathrm{x}-\mathrm{y}+2, \mathrm{z}$ & $\# 3-\mathrm{x}+\mathrm{y}-1 / 3,-\mathrm{x}+4 / 3, \mathrm{z}+1 / 3$ \\
\hline$\# 4 \mathrm{x}-1 / 3, \mathrm{x}-\mathrm{y}+4 / 3, \mathrm{z}+1 / 3$ & $\# 5 \mathrm{x}+1 / 3, \mathrm{y}+2 / 3, \mathrm{z}-1 / 3$ & $\# 6-\mathrm{x}+\mathrm{y}-1 / 3, \mathrm{y}+1 / 3, \mathrm{z}-2 / 3$ \\
\hline$\# 7 \mathrm{x}-1 / 3, \mathrm{y}+1 / 3, \mathrm{z}-2 / 3$ & $\# 8 \mathrm{x}, \mathrm{x}-\mathrm{y}+2, \mathrm{z}$ & $\# 9-\mathrm{x}+\mathrm{y}-2 / 3,-\mathrm{x}+5 / 3, \mathrm{z}-1 / 3$ \\
\hline$\# 10-\mathrm{y}+4 / 3,-\mathrm{x}+5 / 3, \mathrm{z}-1 / 3$ & $\# 11-\mathrm{x}+\mathrm{y}-2 / 3,-\mathrm{x}+2 / 3, \mathrm{z}-1 / 3$ & $\# 12-\mathrm{x}+\mathrm{y}-2 / 3, \mathrm{y}-1 / 3, \mathrm{z}-1 / 3$ \\
\hline$\# 13-\mathrm{y}+4 / 3, \mathrm{x}-\mathrm{y}+5 / 3, \mathrm{z}-1 / 3$ & $\# 14 \mathrm{x}+1 / 3, \mathrm{y}-1 / 3, \mathrm{z}+2 / 3$ & $\# 15-\mathrm{x}+\mathrm{y}, \mathrm{y}, \mathrm{z}$ \\
\hline$\# 16-\mathrm{y}+1, \mathrm{x}-\mathrm{y}+1, \mathrm{z}$ & $\# 17-\mathrm{x}+\mathrm{y},-\mathrm{x}+1, \mathrm{z}$ & $\# 18 \mathrm{x}, \mathrm{x}-\mathrm{y}+1, \mathrm{z}$ \\
\hline$\# 19 \mathrm{x}+2 / 3, \mathrm{y}+1 / 3, \mathrm{z}+1 / 3$ & $\# 20-\mathrm{x}+\mathrm{y}-1 / 3, \mathrm{y}+1 / 3, \mathrm{z}+1 / 3$ & $\# 21-\mathrm{x}+\mathrm{y}-1, \mathrm{y}, \mathrm{z}$ \\
\hline$\# 22-\mathrm{x}+\mathrm{y}-1,-\mathrm{x}+1, \mathrm{z}$ & & \\
\hline
\end{tabular}


Table S4. Selected bond distances $(\AA)$ and angles $(\operatorname{deg})$ for $\mathrm{Rb}_{10} \mathrm{~B}_{13} \mathrm{O}_{15} \mathrm{~F}_{19}$.

\begin{tabular}{|c|c|c|c|}
\hline $\mathrm{Rb}(1)-\mathrm{F}(5 \mathrm{~A}) \# 1$ & $2.68(2)$ & $\mathrm{Rb}(4)-\mathrm{F}(1) \# 14$ & $2.848(18)$ \\
\hline $\mathrm{Rb}(1)-\mathrm{F}(5 \mathrm{~A})$ & $2.68(2)$ & $\mathrm{Rb}(4)-\mathrm{F}(5 \mathrm{~B})$ & $3.05(3)$ \\
\hline $\mathrm{Rb}(1)-\mathrm{O}(2) \# 2$ & $2.734(12)$ & $\mathrm{Rb}(4)-\mathrm{F}(5 \mathrm{~B}) \# 1$ & $3.05(3)$ \\
\hline $\mathrm{Rb}(1)-\mathrm{F}(5 \mathrm{~B}) \# 1$ & $2.834(19)$ & $\mathrm{Rb}(4)-\mathrm{F}(5 \mathrm{~B}) \# 15$ & $3.05(3)$ \\
\hline $\mathrm{Rb}(1)-\mathrm{F}(5 \mathrm{~B})$ & $2.83(2)$ & $\mathrm{Rb}(4)-\mathrm{F}(5 \mathrm{~B}) \# 16$ & $3.05(3)$ \\
\hline $\mathrm{Rb}(1)-\mathrm{O}(3) \# 3$ & $2.948(16)$ & $\mathrm{Rb}(4)-\mathrm{F}(5 \mathrm{~B}) \# 17$ & $3.05(3)$ \\
\hline $\mathrm{Rb}(1)-\mathrm{F}(4) \# 3$ & $3.116(12)$ & $\mathrm{Rb}(4)-\mathrm{F}(5 \mathrm{~B}) \# 18$ & $3.05(3)$ \\
\hline $\mathrm{Rb}(1)-\mathrm{F}(4) \# 4$ & $3.116(12)$ & $\mathrm{Rb}(4)-\mathrm{O}(3)$ & $3.080(14)$ \\
\hline $\mathrm{Rb}(1)-\mathrm{F}(3) \# 2$ & $3.124(7)$ & $\mathrm{Rb}(4)-\mathrm{O}(3) \# 16$ & $3.080(14)$ \\
\hline $\mathrm{Rb}(1)-\mathrm{F}(3)$ & $3.124(7)$ & $\mathrm{Rb}(4)-\mathrm{O}(3) \# 17$ & $3.080(14)$ \\
\hline $\mathrm{Rb}(2)-\mathrm{F}(2) \# 5$ & $2.698(12)$ & $\mathrm{B}(1)-\mathrm{O}(2) \# 2$ & $1.415(11)$ \\
\hline $\mathrm{Rb}(2)-\mathrm{F}(5 \mathrm{~B}) \# 6$ & $2.857(18)$ & $\mathrm{B}(1)-\mathrm{O}(2)$ & $1.415(11)$ \\
\hline $\mathrm{Rb}(2)-\mathrm{F}(5 \mathrm{~B}) \# 7$ & $2.857(18)$ & $\mathrm{B}(1)-\mathrm{F}(3)$ & $1.44(2)$ \\
\hline $\mathrm{Rb}(2)-\mathrm{F}(5 \mathrm{~A}) \# 6$ & $2.89(2)$ & $\mathrm{B}(1)-\mathrm{F}(2)$ & $1.45(2)$ \\
\hline $\mathrm{Rb}(2)-\mathrm{F}(5 \mathrm{~A}) \# 7$ & $2.89(2)$ & $\mathrm{B}(2)-\mathrm{O}(3)$ & $1.38(2)$ \\
\hline $\mathrm{Rb}(2)-\mathrm{O}(1) \# 2$ & $2.932(16)$ & $\mathrm{B}(2)-\mathrm{F}(5 \mathrm{~A})$ & $1.37(3)$ \\
\hline $\mathrm{Rb}(2)-\mathrm{O}(1) \# 8$ & $2.932(16)$ & $\mathrm{B}(2)-\mathrm{F}(4)$ & $1.42(2)$ \\
\hline $\mathrm{Rb}(2)-\mathrm{O}(4) \# 9$ & $2.967(12)$ & $\mathrm{B}(2)-\mathrm{O}(4)$ & $1.44(2)$ \\
\hline $\mathrm{Rb}(2)-\mathrm{O}(4) \# 10$ & $2.967(12)$ & $\mathrm{B}(2)-\mathrm{F}(5 \mathrm{~B})$ & $1.50(3)$ \\
\hline $\mathrm{Rb}(2)-\mathrm{F}(3) \# 5$ & $3.191(12)$ & $\mathrm{B}(3)-\mathrm{O}(4)$ & $1.374(19)$ \\
\hline $\mathrm{Rb}(2)-\mathrm{F}(1)$ & $3.313(6)$ & $\mathrm{B}(3)-\mathrm{O}(4) \# 15$ & $1.374(19)$ \\
\hline $\mathrm{Rb}(2)-\mathrm{F}(4) \# 10$ & $3.320(14)$ & $\mathrm{B}(3)-\mathrm{O}(1) \# 19$ & $1.47(3)$ \\
\hline $\mathrm{Rb}(3)-\mathrm{F}(4) \# 1$ & $2.868(10)$ & $\mathrm{B}(3)-\mathrm{O}(1) \# 20$ & $1.47(3)$ \\
\hline $\mathrm{Rb}(3)-\mathrm{F}(4)$ & $2.868(10)$ & $\mathrm{B}(4)-\mathrm{O}(1) \# 2$ & $1.423(18)^{*}$ \\
\hline $\mathrm{Rb}(3)-\mathrm{O}(2) \# 2$ & $2.951(13)$ & $\mathrm{B}(4)-\mathrm{O}(1) \# 21$ & $1.423(18)^{*}$ \\
\hline $\mathrm{Rb}(3)-\mathrm{O}(1)$ & $2.967(17)$ & $\mathrm{B}(4)-\mathrm{O}(1) \# 1$ & $1.423(18)^{*}$ \\
\hline $\mathrm{Rb}(3)-\mathrm{O}(1) \# 1$ & $2.967(17)$ & $\mathrm{B}(4)-\mathrm{O}(1) \# 8$ & $1.423(18)^{*}$ \\
\hline $\mathrm{Rb}(3)-\mathrm{F}(2) \# 2$ & $2.973(7)$ & $\mathrm{B}(4)-\mathrm{O}(1) \# 22$ & $1.423(18)^{*}$ \\
\hline $\mathrm{Rb}(3)-\mathrm{F}(2)$ & $2.973(7)$ & $\mathrm{B}(4)-\mathrm{O}(1)$ & $1.423(18) *$ \\
\hline $\mathrm{Rb}(3)-\mathrm{F}(3) \# 11$ & $3.062(11)$ & $\mathrm{B}(4)-\mathrm{F}(1)$ & $1.47(4)$ \\
\hline $\mathrm{Rb}(3)-\mathrm{O}(4) \# 12$ & $3.078(12)$ & $\mathrm{O}(2) \# 2-\mathrm{B}(1)-\mathrm{O}(2)$ & $115.9(18)$ \\
\hline $\mathrm{Rb}(3)-\mathrm{O}(4) \# 13$ & $3.078(12)$ & $\mathrm{O}(2) \# 2-\mathrm{B}(1)-\mathrm{F}(3)$ & $109.7(11)$ \\
\hline $\mathrm{Rb}(3)-\mathrm{F}(5 \mathrm{~A}) \# 12$ & $3.38(4)$ & $\mathrm{O}(2)-\mathrm{B}(1)-\mathrm{F}(3)$ & $109.7(11)$ \\
\hline $\mathrm{Rb}(3)-\mathrm{F}(5 \mathrm{~A}) \# 13$ & $3.38(4)$ & $\mathrm{O}(2) \# 2-\mathrm{B}(1)-\mathrm{F}(2)$ & $108.9(12)$ \\
\hline
\end{tabular}




\begin{tabular}{llll}
\hline $\mathrm{O}(2)-\mathrm{B}(1)-\mathrm{F}(2)$ & $108.9(12)$ & $\mathrm{O}(1) \# 21-\mathrm{B}(4)-\mathrm{O}(1) \# 1$ & $112.7(14)$ \\
$\mathrm{F}(3)-\mathrm{B}(1)-\mathrm{F}(2)$ & $102.9(15)$ & $\mathrm{O}(1) \# 2-\mathrm{B}(4)-\mathrm{O}(1) \# 8$ & $60.7(12)$ \\
$\mathrm{O}(3)-\mathrm{B}(2)-\mathrm{F}(5 \mathrm{~A})$ & $131(2)$ & $\mathrm{O}(1) \# 21-\mathrm{B}(4)-\mathrm{O}(1) \# 8$ & $112.7(14)$ \\
$\mathrm{O}(3)-\mathrm{B}(2)-\mathrm{F}(4)$ & $110.6(16)$ & $\mathrm{O}(1) \# 1-\mathrm{B}(4)-\mathrm{O}(1) \# 8$ & $112.7(14)$ \\
$\mathrm{F}(5 \mathrm{~A})-\mathrm{B}(2)-\mathrm{F}(4)$ & $107.5(15)$ & $\mathrm{O}(1) \# 2-\mathrm{B}(4)-\mathrm{O}(1) \# 22$ & $112.7(14)$ \\
$\mathrm{O}(3)-\mathrm{B}(2)-\mathrm{O}(4)$ & $113.3(14)$ & $\mathrm{O}(1) \# 21-\mathrm{B}(4)-\mathrm{O}(1) \# 22$ & $60.7(12)$ \\
$\mathrm{F}(5 \mathrm{~A})-\mathrm{B}(2)-\mathrm{O}(4)$ & $85(2)$ & $\mathrm{O}(1) \# 1-\mathrm{B}(4)-\mathrm{O}(1) \# 22$ & $148(3)$ \\
$\mathrm{F}(4)-\mathrm{B}(2)-\mathrm{O}(4)$ & $104.4(17)$ & $\mathrm{O}(1) \# 8-\mathrm{B}(4)-\mathrm{O}(1) \# 22$ & $54.2(12)$ \\
$\mathrm{O}(3)-\mathrm{B}(2)-\mathrm{F}(5 \mathrm{~B})$ & $98(2)$ & $\mathrm{O}(1) \# 2-\mathrm{B}(4)-\mathrm{O}(1)$ & $112.7(14)$ \\
$\mathrm{F}(4)-\mathrm{B}(2)-\mathrm{F}(5 \mathrm{~B})$ & $107.1(14)$ & $\mathrm{O}(1) \# 21-\mathrm{B}(4)-\mathrm{O}(1)$ & $54.2(12)$ \\
$\mathrm{O}(4)-\mathrm{B}(2)-\mathrm{F}(5 \mathrm{~B})$ & $123.0(19)$ & $\mathrm{O}(1) \# 1-\mathrm{B}(4)-\mathrm{O}(1)$ & $60.7(12)$ \\
$\mathrm{O}(4)-\mathrm{B}(3)-\mathrm{O}(4) \# 15$ & $117(2)$ & $\mathrm{O}(1) \# 8-\mathrm{B}(4)-\mathrm{O}(1)$ & $148(3)$ \\
$\mathrm{O}(4)-\mathrm{B}(3)-\mathrm{O}(1) \# 19$ & $147.6(19)$ & $\mathrm{O}(1) \# 22-\mathrm{B}(4)-\mathrm{O}(1)$ & $112.7(14)$ \\
$\mathrm{O}(4) \# 15-\mathrm{B}(3)-\mathrm{O}(1) \# 19$ & $95.1(9)$ & $\mathrm{O}(1) \# 2-\mathrm{B}(4)-\mathrm{F}(1)$ & $106.0(16)$ \\
$\mathrm{O}(4)-\mathrm{B}(3)-\mathrm{O}(1) \# 20$ & $95.1(9)$ & $\mathrm{O}(1) \# 21-\mathrm{B}(4)-\mathrm{F}(1)$ & $106.0(16)$ \\
$\mathrm{O}(4) \# 15-\mathrm{B}(3)-\mathrm{O}(1) \# 20$ & $147.6(19)$ & $\mathrm{O}(1) \# 1-\mathrm{B}(4)-\mathrm{F}(1)$ & $106.0(16)$ \\
$\mathrm{O}(1) \# 19-\mathrm{B}(3)-\mathrm{O}(1) \# 20$ & $52.5(16)$ & $\mathrm{O}(1) \# 8-\mathrm{B}(4)-\mathrm{F}(1)$ & $106.0(16)$ \\
$\mathrm{O}(1) \# 2-\mathrm{B}(4)-\mathrm{O}(1) \# 21$ & $148(3)$ & $\mathrm{O}(1) \# 22-\mathrm{B}(4)-\mathrm{F}(1)$ & $106.0(16)$ \\
$\mathrm{O}(1) \# 2-\mathrm{B}(4)-\mathrm{O}(1) \# 1$ & $54.2(12)$ & $\mathrm{O}(1)-\mathrm{B}(4)-\mathrm{F}(1)$ & $106.0(16)$ \\
\hline
\end{tabular}

*: The $\mathrm{O}(1)$ is disordered in special site.

Symmetry transformations used to generate equivalent atoms:

\begin{tabular}{|l|l|l|}
\hline$\# 1-y+1,-x+1, z$ & $\# 2-y+1, x-y+2, z$ & $\# 3-x+y-1 / 3,-x+4 / 3, z+1 / 3$ \\
\hline$\# 4 x-1 / 3, x-y+4 / 3, z+1 / 3$ & $\# 5 x+1 / 3, y+2 / 3, z-1 / 3$ & $\# 6-x+y-1 / 3, y+1 / 3, z-2 / 3$ \\
\hline$\# 7 x-1 / 3, y+1 / 3, z-2 / 3$ & $\# 8 x, x-y+2, z$ & $\# 9-x+y-2 / 3,-x+5 / 3, z-1 / 3$ \\
\hline$\# 10-y+4 / 3,-x+5 / 3, z-1 / 3$ & $\# 11-x+y-2 / 3,-x+2 / 3, z-1 / 3$ & $\# 12-x+y-2 / 3, y-1 / 3, z-1 / 3$ \\
\hline$\# 13-y+4 / 3, x-y+5 / 3, z-1 / 3$ & $\# 14 x+1 / 3, y-1 / 3, z+2 / 3$ & $\# 15-x+y, y, z$ \\
\hline$\# 16-y+1, x-y+1, z$ & $\# 17-x+y,-x+1, z$ & $\# 18 x, x-y+1, z$ \\
\hline$\# 19 x+2 / 3, y+1 / 3, z+1 / 3$ & $\# 20-x+y-1 / 3, y+1 / 3, z+1 / 3$ & $\# 21-x+y-1, y, z$ \\
\hline$\# 22-x+y-1,-x+1, z$ & & \\
\hline
\end{tabular}



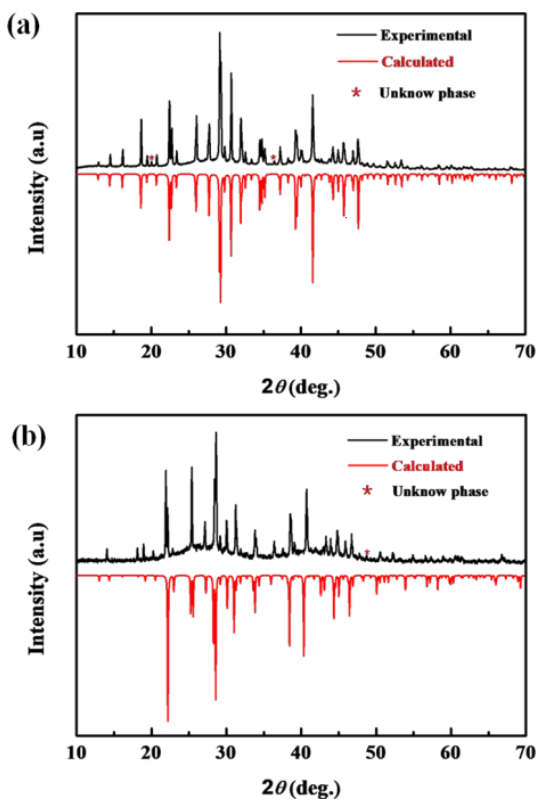

Figure S1 Calculated and experimental powder X-ray diffraction patterns for (a) $\mathrm{K}_{10} \mathrm{~B}_{13} \mathrm{O}_{15} \mathrm{~F}_{19}$, (b)

$$
\mathrm{Rb}_{10} \mathrm{~B}_{13} \mathrm{O}_{15} \mathrm{~F}_{19}
$$




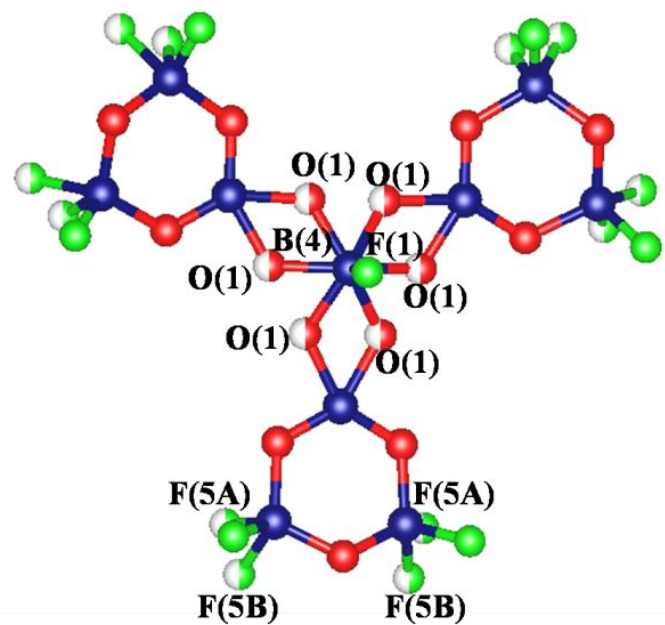

Figure S2 The final refined site occupation factor of $F(5)$ is $0.5: 0.5$ and the $\mathrm{O}(1)$ atoms in the motif of $\left[\mathrm{B}_{10} \mathrm{O}_{12} \mathrm{~F}_{13}\right]^{7-}$ is located at the special positions of site occupation factor 0.5 . 

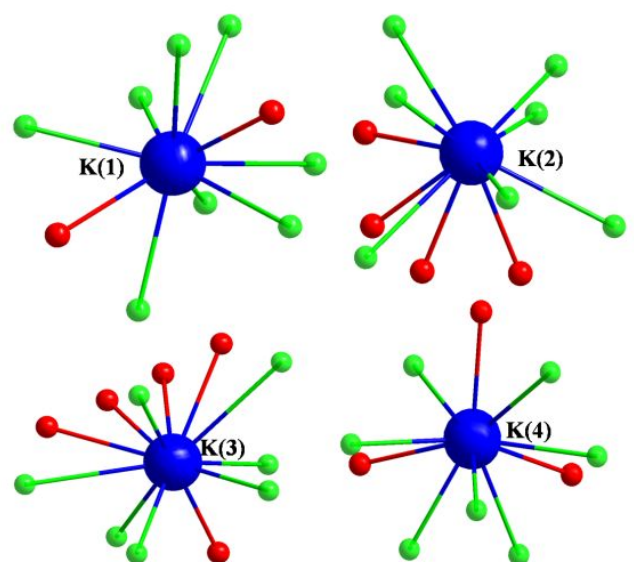

Figure S3 The coordination environment of the $\mathrm{K}^{+}$cations. 

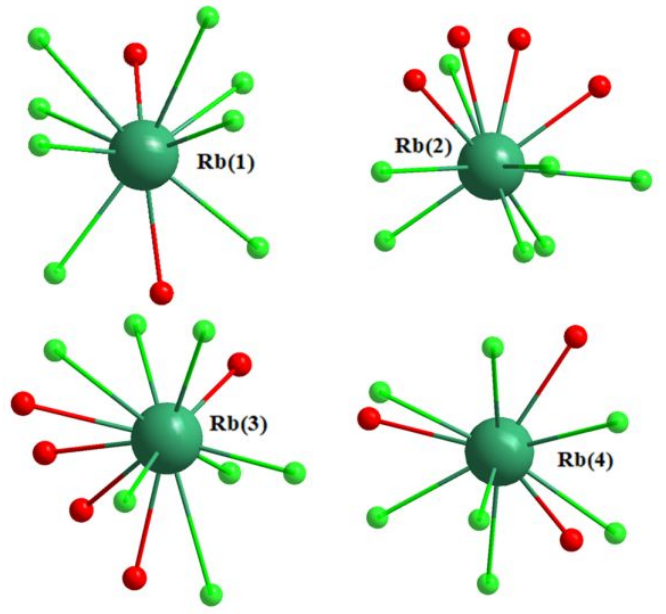

Figure $\mathrm{S} 4$ The coordination environment of the $\mathrm{Rb}^{+}$cations. 
(a)

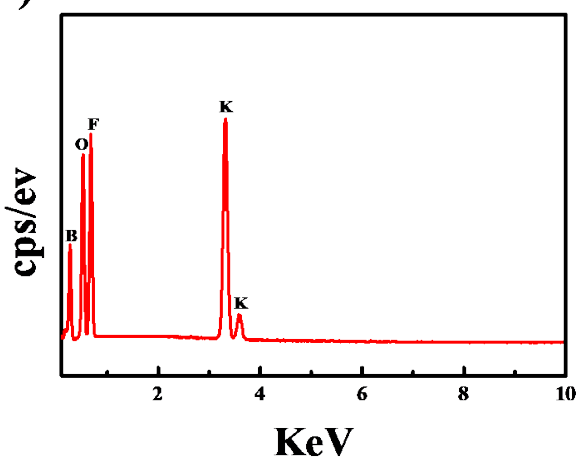

(b)

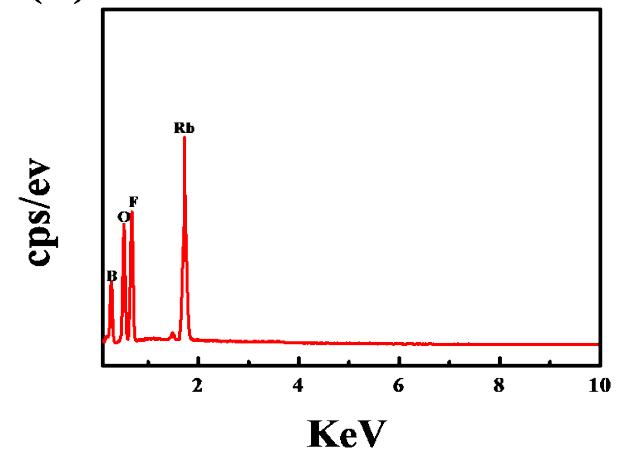

Figure S5 The EDX spectra of the as-synthesized (a) $\mathrm{K}_{10} \mathrm{~B}_{13} \mathrm{O}_{15} \mathrm{~F}_{19}$ and (b) $\mathrm{Rb}_{10} \mathrm{~B}_{13} \mathrm{O}_{15} \mathrm{~F}_{19}$. 
(a)

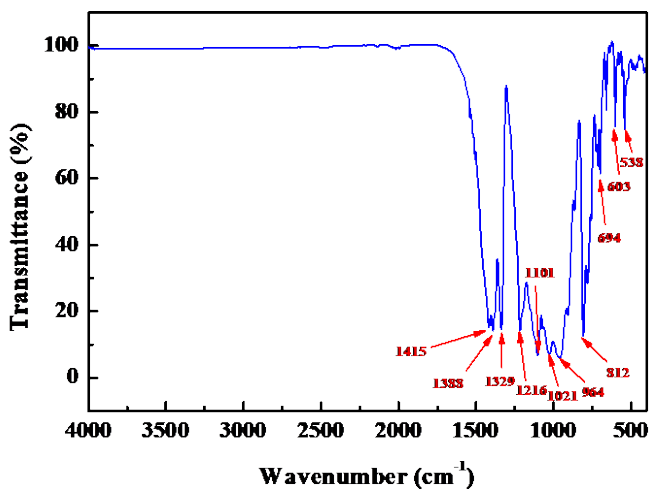

(b)

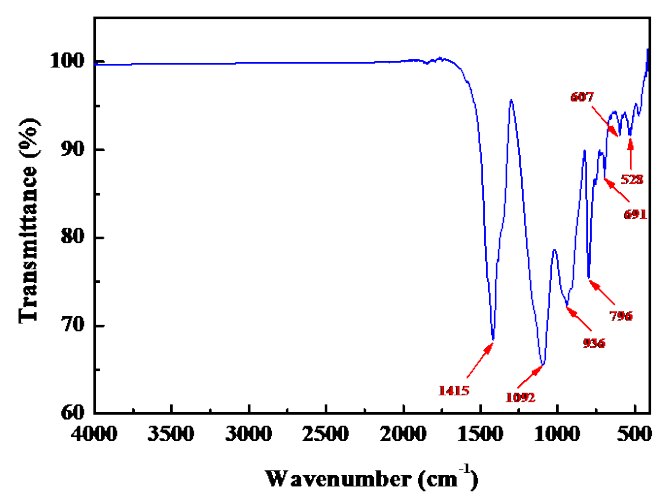

Figure S6 IR spectra for (a) $\mathrm{K}_{10} \mathrm{~B}_{13} \mathrm{O}_{15} \mathrm{~F}_{19}$, (b) $\mathrm{Rb}_{10} \mathrm{~B}_{13} \mathrm{O}_{15} \mathrm{~F}_{19}$. 
1. Wang, Y.; Zhang, B. B.; Yang, Z. H.; Pan, S. L., Cation-Tuned Synthesis of Fluorooxoborates: Towards Optimal Deep-Ultraviolet Nonlinear Optical Materials. Angew. Chem. Int. Ed. 2018, 57, 2150-2154. 